\title{
Inhibition of aerobic glycolysis alleviates sepsis-induced acute kidney injury by promoting lactate/Sirtuin 3/AMPK-regulated autophagy
}

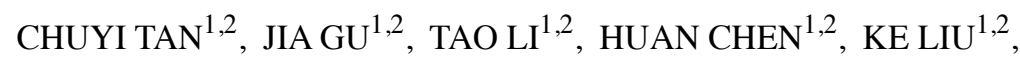 \\ MEIDONG LIU ${ }^{1,2}$, HUALI ZHANG ${ }^{1,2^{*}}$ and XIANZHONG XIAO ${ }^{1,2^{*}}$ \\ ${ }^{1}$ Department of Pathophysiology, Xiangya School of Medicine, Central South University; \\ ${ }^{2}$ Sepsis Translational Medicine Key Laboratory of Hunan Province, \\ Central South University, Changsha, Hunan 410078, P.R. China
}

Received June 2, 2020; Accepted November 16, 2020

DOI: $10.3892 /$ ijmm.2021.4852

\begin{abstract}
Metabolism reprogramming influences the severity of organ dysfunction, progression to fibrosis, and development of disease in acute kidney injury (AKI). Previously we showed that inhibition of aerobic glycolysis improved survival rates and protected septic mice from kidney injury. However, the underlying mechanisms remain unclear. In the present study, it was revealed that sepsis or lipopolysaccharide (LPS) enhanced aerobic glycolysis as evidenced by increased lactate production and upregulated mRNA expression of glycolysis-related genes in kidney tissues and human renal tubular epithelial (HK-2) cells. The aerobic glycolysis inhibitor 2-deoxy-D-glucose (2-DG) downregulated glycolysis, and improved kidney injury induced by sepsis. 2-DG treatments increased the expression of
\end{abstract}

Correspondence to: Professor Huali Zhang or Professor Xianzhong Xiao, Department of Pathophysiology, Xiangya School of Medicine, Central South University, 110 Xiangya Road, Kaifu, Changsha, Hunan 410078, P.R. China

E-mail: zhanghuali@csu.edu.cn

E-mail: xiaoxianzhong@csu.edu.cn

*Joint senior authorship

Abbreviations: 2-DG, 2-deoxy-D-glucose; AKI, acute kidney injury; AMPK, AMP-activated protein kinase; BUN, blood urea nitrogen; CLP, cecal ligation and puncture; LDHA, lactate dehydrogenase A; LPS, lipopolysaccharides; mTOR, mammalian target of rapamycin; PDK1, pyruvate dehydrogenase kinase 1; PKM2, pyruvate kinase M2; Scr, serum creatinine; SIRT3, sirtuin 3; 3-MA, 3-methyladenine; PBS, phosphate-buffered saline; DAPI, 4,6-diamino-2-phenyl indole; LC3, microtubule-associated protein light chain 3; HK-2, human renal proximal tubular epithelial cell line; DMEM, Dulbecco's modified Eagle's medium; FITC, fluorescein isothiocyanate; RIPA, radio-immunoprecipitation

Key words: aerobic glycolysis, 2-deoxy-D-glucose, autophagy, acute kidney injury, sepsis sirtuin 3 (SIRT3) and phosphorylation-AMP-activated protein kinase (p-AMPK), following promoted autophagy and attenuated apoptosis of tubular epithelial cells in septic mice and in LPS-treated HK-2 cells. However, the glycolysis metabolite lactate downregulated SIRT3 and p-AMPK expression, inhibited autophagy and enhanced apoptosis in LPS-treated HK-2 cells. Furthermore, pharmacological blockade of autophagy with 3-methyladenine (3-MA) partially abolished the protective effect of 2-DG in sepsis-induced AKI. These findings indicated that inhibition of aerobic glycolysis protected against sepsis-induced AKI by promoting autophagy via the lactate/SIRT3/AMPK pathway.

\section{Introduction}

Acute kidney injury (AKI) is a serious and common complication of sepsis (1), and is also a risk factor for progression to chronic kidney disease (2). Substantial evidence indicates that patients who are diagnosed with septic AKI have a higher mortality risk and generally have longer ICU and hospital stays than patients with non-septic AKI $(3,4)$. Unfortunately, as largely supportive therapies of sepsis-induced AKI are often ineffective, the mortality related to AKI remains high. Therefore, efforts focused on clarifying the specific pathogenesis are important for finding a new therapy for sepsis-induced AKI.

In recent years, evidence has demonstrated that autophagy has a protective role in AKI $(5,6)$. For instance, numerous studies have revealed that autophagy is activated in renal tubular epithelial cells upon the occurrence of AKI (7), and blockade of autophagy pharmacologically or genetically leads to more severe AKI, while induction of autophagy reduces kidney injury $(8,9)$. However, the regulatory mechanism of autophagy in AKI is complicated and has not been fully elucidated.

Increasing evidence suggests that metabolic reprogramming has a central role in the immune response of host defense to infection and represents a novel target for inflammatory diseases. Early metabolic reprogramming in sepsis-induced AKI not only protects the kidney from further injury, but 
also determines the fate of tissue repair and the progression to fibrosis and chronic organ dysfunction $(10,11)$. Smith et al demonstrated a shift in metabolism towards aerobic glycolysis in mouse kidney tissues after administration with lipopolysaccharide (LPS), which is associated with a decline in renal function (12). In cecal ligation and puncture (CLP)-induced septic mice, metabolomic analysis of kidney tissues revealed increases in the levels of glycolytic intermediates (13). In addition, growing evidence indicates that autophagy regulates or controls metabolism, which in turn affects autophagy (14). For instance, the knockdown of the key glycolysis enzyme pyruvate kinase M2 (PKM2) decreased glycolysis and induced autophagy in human A549 alveolar adenocarcinoma cells (15). Furthermore, our previous work revealed that inhibition of aerobic glycolysis improved survival rates and protected septic mice from kidney injury (16). However, the mechanism of the protective effect of inhibiting aerobic glycolysis on sepsis-induced AKI is unknown, and whether this protective effect is achieved by regulating autophagy in sepsis-induced $\mathrm{AKI}$ is also unknown.

The aim of the present study, was to study the exact mechanism by which aerobic glycolysis regulates autophagy in sepsis-induced AKI. It was hypothesized that aerobic glycolysis contributes to modulating autophagy via the lactate/sirtuin 3 (SIRT3)/AMP-activated protein kinase (AMPK) pathway in CLP-induced septic AKI. Pharmacological inhibition of glycolysis by 2-deoxy-D-glucose (2-DG) and AKI was induced by CLP in BALB/C mice in the present study.

\section{Materials and methods}

Mice. In total 60 BALB/c mice (male; 6-8 weeks old; 20-25 g) were purchased from Hunan SJA Laboratory Animal Co., Ltd. All mice were housed individually and were maintained at $22^{\circ} \mathrm{C}$ with $40-60 \%$ humidity and a 12 -h light/dark cycle starting at 6:00 am. The mice were fed with normal chow and had free access to food and water throughout the experiment. All animal experimental protocols were approved by the Institutional Animal Care and Use Committee of Central South University (approval no. 2019sydw0027) and performed according to the guidelines of the Ethics Committee of the Animal Experimental Institute of Central South University, Changsha, China.

Cecal ligation and puncture (CLP)-induced sepsis. Compared to LPS-induced sepsis, the CLP-induced septic model is closer to human sepsis since it is characterized by a polymicrobial insult, hyper-then hypodynamic hemodynamic transition, the generation of detectable bacteremia, and the increase in damage-associated molecular patterns. Therefore, CLP was selected to induce sepsis.

Sepsis was induced in BALB/c mice by CLP as previously described (17). The mice were anaesthetized by isoflurane and fixed on the table. A middle incision of the belly was made and the abdomen was opened. Then, the cecum was exposed and ligated. Two punctures were produced with a 22-gauge needle at the end of the cecum and some feces were extruded from the punctures. The cecum was turned back inside the abdomen, and the abdomen was closed. Resuscitative fluid (normal saline, $50 \mathrm{ml} / \mathrm{kg}$ ) was administrated by subcutaneous injection. All operations were the same in the sham group except for no puncture and ligation of the cecum.

Animal treatment. From the 60 mice, 24 mice were randomly divided into four groups: The sham group, the sham+2-DG group, the CLP group, and the CLP+2-DG group. The glycolysis inhibitor 2-DG (Sigma-Aldrich; Merck KGaA) was dissolved in sterile saline and injected intraperitoneally ( $2 \mathrm{~g} / \mathrm{kg}$ ) $3 \mathrm{~h}$ before the CLP operation. An equal volume of vehicle was intraperitoneally injected as a control. The dose and time-point of 2-DG was selected based on previous studies $(16,18)$.

From the 60 mice, 36 mice were randomly divided into six groups: The sham group, the sham+2-DG group, the CLP group, the CLP+2-DG group, the CLP+3-methyladenine (3-MA) group, and the CLP+2-DG+3-MA group. Autophagy inhibitor 3-MA (Selleck Chemicals) was dissolved in PBS by heating and injected intraperitoneally $(30 \mathrm{mg} / \mathrm{kg}) 30 \mathrm{~min}$ before the CLP operation. An equal volume of vehicle was intraperitoneally injected as a control. The dose and time-point of 3-MA were selected based on previous studies (19).

At $24 \mathrm{~h}$ of the CLP or sham operation, serum and kidney tissues were harvested from mice and stored at $-80^{\circ} \mathrm{C}$ for the following experiments.

Haematoxylin and eosin $(H \& E)$ dye and transferase terminal UTP nick-end labeling (TUNEL) staining. Kidney sections were fixed in $10 \%$ buffered formalin at room temperature for $24 \mathrm{~h}$, embedded in paraffin, cut into $3-\mu \mathrm{m}$ sections, stained with $H \& E$ at room temperature for $3 \mathrm{~min}$, and visualized at a magnification of $\mathrm{x} 400$ under an optical microscope (Olympus Optical Co., Ltd.).

Apoptosis of kidney tissue was detected using the TUNEL assay (Roche Diagnostics) according to the manufacturer's instructions. The renal tissues $(4 \mu \mathrm{m})$ were fixed in $10 \%$ buffered formalin at room temperature for $24 \mathrm{~h}$, paraffin-embedded and labeled with TUNEL reaction mixture containing terminal deoxynucleotidyl transferase and nucleotides including tetramethylrhodamine labeled (TMR-labeled) dUTP at room temperature for $1 \mathrm{~h}$. DAPI was then used to stain the nucleus at room temperature for $15 \mathrm{~min}$ followed by the addition of a fluorescent mounting medium. The TUNEL-positive cells were counted in 5 randomly selected fields from each slide at a magnification of $x 400$, and the percentage of TUNEL-positive cells was calculated from four kidney sections of different mice.

Cell treatment in vitro. A human renal proximal tubular epithelial cell line (HK-2 cells) purchased from ATCC was cultured in DMEM/F12 medium supplemented with $10 \%$ fetal bovine serum at $37^{\circ} \mathrm{C}$ in an incubator supplemented with $5 \% \mathrm{CO}_{2}$. HK-2 cells $\left(1 \times 10^{6}\right)$ were plated into 6 -well plates, and pre-treated with or without 2-DG ( $2 \mathrm{mM}$, before $3 \mathrm{~h}$ ) or/and 3-MA ( $5 \mathrm{mM}$, before $1 \mathrm{~h})$ and/or lactate $(25 \mu \mathrm{M})$, followed by stimulation with or without LPS $(1 \mu \mathrm{g} / \mathrm{ml})$ for $12 \mathrm{~h}$. Lactate was added to the culture medium of HK-2 cells without adjustment of $\mathrm{pH}$. It was determined that the extracellular $\mathrm{pH}$ changed from 7.2 to 6.6 after the addition of lactate in LPS-stimulated HK-2 cells. Then cells were harvested and protein and total RNA were isolated for subsequent protein and RNA studies, and the culture medium was collected and stored at $-20^{\circ} \mathrm{C}$. 
Table I. Primers used for qPCR in the present study.

\begin{tabular}{|c|c|c|}
\hline Primer names & Forward (5'-3') & Reverse (5'-3') \\
\hline Human-PKM2 & \multirow[t]{2}{*}{ ATTATTTGAGGAACTCCGCCGCCT } & \multirow[t]{2}{*}{ ATTCCGGGTCACAGCAATGATGG } \\
\hline NM002654.6 & & \\
\hline Human-LDHA & \multirow[t]{2}{*}{ ATCTTGACCTACGTGGCTTGGA } & \multirow[t]{2}{*}{ CCATACAGGCACACTGGAATCTC } \\
\hline NM005566.4 & & \\
\hline Human-PDK1 & \multirow[t]{2}{*}{ ACCAGGACAGCCAATACAAG } & \multirow[t]{2}{*}{ CCTCGGTCACTCATCTTCAC } \\
\hline NM002610.5 & & \\
\hline Human-18S & \multirow[t]{2}{*}{ ATCCTCAGTGAGTTCTCCCG } & \multirow[t]{2}{*}{ CTTTGCCATCACTGCCATTA } \\
\hline NM022551.3 & & \\
\hline Mouse- PKM2 & \multirow[t]{2}{*}{ TGTCTGGAGAAACAGCCAAG } & \multirow[t]{2}{*}{ TCCTCGAATAGCTGCAAGTG } \\
\hline NM011099.3 & & \\
\hline Mouse-LDHA & \multirow[t]{2}{*}{ AGAGCGGGAGGGCAGCTTTCT } & \multirow[t]{2}{*}{ GGGCAAGCTCATCCGCCAAGT } \\
\hline NM001136069.2 & & \\
\hline Mouse-PDK1 & \multirow[t]{2}{*}{ TTCTCGCCGTCGCCACTCTC } & \multirow[t]{2}{*}{ TGTCGGGGAGGAGGCTGATTTC } \\
\hline NM001360002.1 & & \\
\hline Mouse-actin & \multirow[t]{2}{*}{ AGAAAATCTGGCACCACACC } & \multirow[t]{2}{*}{ CAGAGGCGTACAGGGATAGC } \\
\hline NM205518.1 & & \\
\hline
\end{tabular}

PKM2, pyruvate kinase M2; LDHA, lactate dehydrogenase A; PDK1, pyruvate dehydrogenase kinase 1.

Measurement of lactate. The lactate concentration of serum and culture medium was measured using a Lactate Assay kit (cat. no. A019-2-1; Nanjing Jiancheng Bioengineering Institute), according to the manufacturer's instructions. The soluble fraction was assayed directly at $530 \mathrm{~nm}$ using ELX-800 absorbance microplate reader (BioTek Instruments, Inc.) until completion of the reaction. Before the sample was analyzed, several samples we tested to determine the applicable loading volume, which ensures the readings within the standard curve. Lactate contents in the samples were calculated according to the corresponding formula: Lactate $(\mathrm{mmol} / \mathrm{l})=($ sample OD-blank OD)/(standard OD-blank OD) x standard concentration $(\mathrm{mmol} / \mathrm{l}) \mathrm{x}$ diluent times.

Biochemical assays. The sera of mice were collected at the indicated time-points. Commercially available blood urea nitrogen (BUN) and serum creatinine (Scr) detection kits (cat. nos. C010 and C074; Changchun Huili Biotech Co., Ltd.) were used to measure serum levels of biochemical parameters including blood urea nitrogen (BUN) and serum creatinine (Scr), according to the manufacturer's instructions, using an Olympus AU5400 Automated Biochemical Analyzer (Olympus Corporation). The serum concentrations of kidney injury molecular-1 (KIM-1) were measured with an ELISA kit (cat. no. EK0880; BOSTER Biological Technology Co., Ltd.) according to the manufacturer's instructions.

Apoptosis detection using flow cytometry. Cell apoptosis analysis using the Annexin V-FITC/PI Apoptosis Detection kit (BD Biosciences) was measured by flow cytometry according to the manufacturer's instructions. After stimulation, $1.0 \times 10^{6} \mathrm{HK}-2$ cells were washed with cold PBS. Then, Annexin V-FITC and propidium iodide (PI) staining was performed in the dark at room temperature for $15 \mathrm{~min}$. Apototic cells were detected by flow cytometry using a BD FACSCantoII flow cytometer (BD Biosciences) and were analyzed with FlowJo analytical software (Version X; TreeStar, Inc.).

Reverse transcription-quantitative $(R T-q) P C R$ analysis. Total cell or tissue RNA was extracted with TRIzol Reagent (Invitrogen; Thermo Fisher Scientific, Inc.) and quantitated by Nanodrop spectrophotometry. Reverse transcription was performed with a PrimeScript ${ }^{\mathrm{TM}}$ RT Reagent kit according to the manufacturer's instructions (Takara Bio, Inc.). The cDNAs were subjected to quantitative real-time PCR using gene-specific primers (300 $\mathrm{nM}$ per reaction) according to TB Green $^{\mathrm{TM}}$ Premix Ex Taq ${ }^{\mathrm{TM}}$ (Takara Bio, Inc.) protocols. The amplification level was programmed with a denaturation step of 3 min at $95^{\circ} \mathrm{C}$, followed by 40 cycles of denaturation at $95^{\circ} \mathrm{C}$ for $12 \mathrm{sec}$, and extension at $62^{\circ} \mathrm{C}$ for $40 \mathrm{sec}$. Human- $18 \mathrm{~S}$ ribosomal and mouse-actin mRNA levels served as internal controls. Data were calculated using the $2^{-\Delta \Delta C q}$ method method (20). The primers used in the present study are presented in Table I.

Western blotting. After stimulation, HK-2 cells were washed twice with ice-cold PBS and lysed by RIPA (Thermo Fisher Scientific, Inc.) with phosphatase and protein inhibitor for $30 \mathrm{~min}$ on ice, followed by sonication twice. Then whole cell lysate was centrifuged at $4^{\circ} \mathrm{C}$, and $16,000 \times \mathrm{g}$ for $10 \mathrm{~min}$. The supernatants were collected for proteins analysis. Protein concentration in the supernatants was determined using the bicinchoninic acid (BCA) protein assay. Cell proteins $(10 \mu \mathrm{g})$ were separated by $4-12 \%$ sodium dodecyl sulfate-polyacrylamide gel electrophoresis and transferred to a polyvinylidene fluoride membrane. The membrane was blocked in 5\% milk at room temperature for $2 \mathrm{~h}$ and then were incubated in primary antibodies against LC3-I/II (product no. ABC929; Sigma-Aldrich; Merck KGaA), SIRT3 (product no. 5490S), 
phosphorylated (p)-AMPK $\alpha$ (Thr172) (product no. 2535S), AMPK $\alpha$ (product no. 2603S), p62 (product no. 5114S) and $\beta$-actin (product no. 3700S) (all 1:1,000; from Cell Signaling Technology, Inc.) at $4^{\circ} \mathrm{C}$ overnight. Membranes were washed in TBS-T (0.01\% Tween) 3 times and incubated with HRP-conjugated secondary antibody (goat anti-mouse; cat. no. BA1050 and goat anti-rabbit, cat. no. BA1054 both from BOSTER Biological Technology co. ltd.) at room temperature for $1 \mathrm{~h}$. The signals were visualized with ECL substrate (Bio-Rad Laboratories, Inc.) and quantified using ImageJ $1.48 \mathrm{~V}$ software (NIH).

Immunofluorescence staining. HK-2 cells $\left(1.0 \times 10^{6}\right)$ were washed with ice-cold PBS twice and fixed with $4 \%$ methanol for $15 \mathrm{~min}$ at room temperature. Then the cells were washed with PBS three times and blocked with 5\% PBS-BSA at room temperature for $1 \mathrm{~h}$. Coverslips were incubated with primary antibody against LC3 (dilution 1:200; product no. L8918; Sigma-Aldrich; Merck KGaA) overnight at $4^{\circ} \mathrm{C}$. FITC goat anti-rabbit IgG (dilution 1:200; cat. no. AS011; ABclonal Biotech Co., Ltd.) served as the secondary antibody at room temperature for $1 \mathrm{~h}$. DAPI was then used to stain the nucleus at room temperature for $15 \mathrm{~min}$, followed by the addition of a fluorescent mounting medium. Cells were visualized at a magnification of $\mathrm{x} 400$ using fluorescence microscopy (Olympus BX53; Olympus Corporation.).

Statistical analysis. All statistical analyses were performed with GraphPad Prism version 7.0 software (GraphPad Software, Inc.). Comparisons between two groups were performed with either an unpaired two-tailed Student's t-test (parametric) or the Mann-Whitney test (non-parametric). Comparisons between multiple groups were analyzed using one-way analysis of variance, followed by Tukey's multiple comparison test. A P-value $<0.05$ was considered to indicate a statistically significant difference.

\section{Results}

Aerobic glycolysis is upregulated in CLP-induced AKI. First, the mRNA levels of glycolysis-related genes and the glycolysis metabolite lactate in vivo were detected to verify that glycolysis is upregulated in sepsis-induced AKI. In fact, it was revealed that the mRNA expression levels of glycolysis-related genes pyruvate dehydrogenase kinase 1 (PDK1), lactate dehydrogenase A (LDHA), and pyruvate kinase M2 (PKM2) were upregulated in the kidney tissue of septic mice compared to those in the sham group. In contrast, inhibition of aerobic glycolysis by 2-DG downregulated the mRNA expression levels of PDK1, LDHA, and PKM2 in the kidney tissue of septic mice (Fig. 1A-C). Moreover, the concentration of serum lactate was increased in septic mice, while it was decreased in septic mice by treatment with 2-DG (Fig. 1D). These results indicated that aerobic glycolysis was upregulated in sepsis-induced AKI, which could be reversed by the glycolysis inhibitor 2-DG.

Aerobic glycolysis inhibitor 2-DG alleviates sepsis-induced $A K I$. To assess the effect of aerobic glycolysis on the kidney function of septic mice, we assessed the serum levels of BUN, creatinine and KIM-1 in CLP-induced septic mice. Mice were treated with 2-DG $(2 \mathrm{~g} / \mathrm{kg})$ or vehicle $(0.9 \% \mathrm{NaCl})$ before CLP $3 \mathrm{~h}$. The dose of 2-DG administered was established by our previous work, which improved the survival rates of septic mice (16). In the present study, the serum levels of BUN, creatinine and KIM-1 were increased in septic mice compared to those in the sham group, and this increase was significantly decreased by 2-DG (Fig. 2A-C). In addition, the morphological tubular $\mathrm{H} \& \mathrm{E}$ dye revealed that the tubular epithelial cells of septic mice were edematous with larger cellular volume, vacuolar degeneration and glomerular structure was disordered accompanied by narrowed lumen of the renal tubules. These pathological lesions were alleviated by 2-DG treatment in the septic mice (Fig. 2D). These results indicated that inhibition of aerobic glycolysis has a protective effect on sepsis-induced AKI.

Aerobic glycolysis inhibitor 2-DG alleviates sepsis-induced AKI by upregulating autophagy. Next, the role of autophagy in the protective effect of the aerobic glycolysis inhibitor 2-DG against sepsis-induced AKI was investigated. Western blotting revealed that CLP upregulated autophagy as evidenced by the increase of the ratio of LC3-II/I and decrease of the expression of p62 compared to the sham group, and this effect was further improved by treatment with 2-DG in septic mice (Fig. 3A and B). In addition, it has been established that autophagy always interacts with apoptosis (21). In the present study it was revealed that CLP enhanced the apoptosis of renal tubular epithelial cells compared to the sham group, and this increase was significantly suppressed by 2-DG in septic mice treated with 2-DG (Fig. 3C). These results indicated that 2-DG-mediated protection against sepsis-induced AKI was related to an increased regulation of autophagy.

Aerobic glycolysis is upregulated in $\mathrm{HK}-2$ cells stimulated with LPS. The mRNA levels of glycolysis-related genes and the glycolysis metabolite lactate were also detected in vitro in HK-2 cells stimulated with LPS. It was revealed that the mRNA expression of LDHA and PKM2 was upregulated in HK-2 cells stimulated with LPS compared to those in the control group. In contrast, 2-DG decreased the mRNA expression of LDHA and PKM2 in HK-2 cells stimulated with LPS (Fig. 4B and C). The mRNA expression of PDK1 was unchanged in HK-2 cells (Fig. 4A). Moreover, LPS enhanced the lactate levels in the culture supernatants of HK-2 cells compared to the control group, while 2-DG reduced the lactate levels in the culture supernatant of HK-2 cells stimulated with LPS (Fig. 4D). These results indicated that aerobic glycolysis was upregulated in HK-2 cells stimulated with LPS, which could be reversed by the glycolysis inhibitor 2-DG.

Aerobic glycolysis inhibitor 2-DG further upregulates autophagy in HK-2 cells stimulated with LPS. The relationship between autophagy and aerobic glycolysis in vitro in HK-2 cells stimulated with LPS was also investigated. It was observed that LPS enhanced the ratio of LC3-II/I and decreased p62 expression compared to those in the control group, and this effect was further promoted by 2-DG (Fig. 5A). Immunofluorescence also revealed that LPS enhanced the expression of LC3-II, and this effect was further improved by 
A
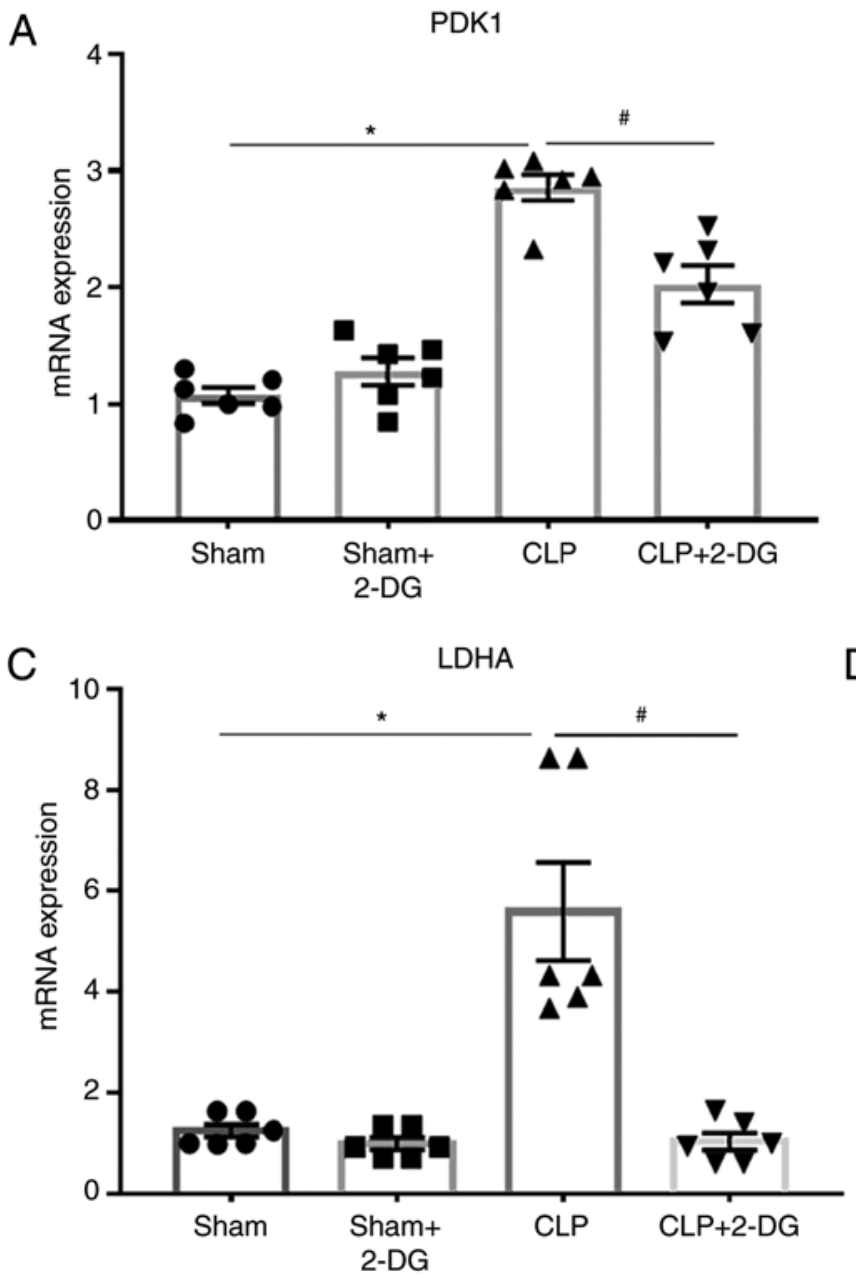

B

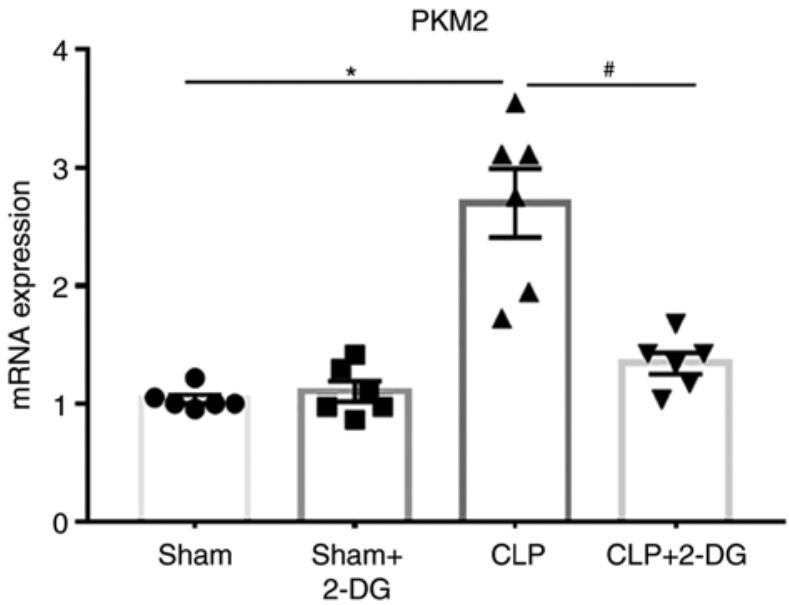

D

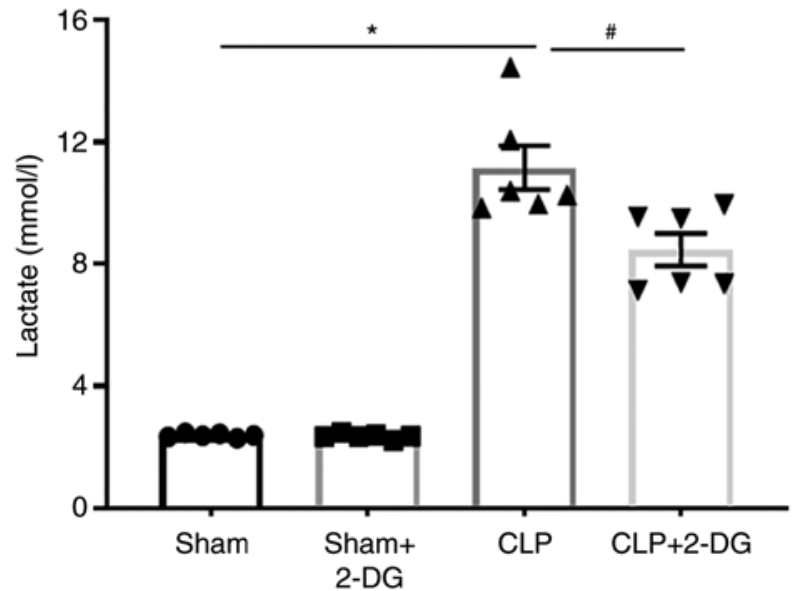

Figure 1. Assessment of glycolysis in kidney tissues of CLP-induced septic mice. Sepsis was induced in BALB/c mice by CLP. BALB/c mice were injected i.p. with either 2-DG $(2 \mathrm{~g} / \mathrm{kg})$ or PBS in equivalent volumes $3 \mathrm{~h}$ before CLP. Sham-operated animals served as negative controls. At $24 \mathrm{~h}$ of CLP or sham operation, serum and kidney were harvested from mice. (A-C) The mRNA expression levels of glycolysis-related genes (PDK1, PKM2 and LDHA) were analyzed by real-time PCR in the kidney tissues of mice. $n=6$ mice per group. (D) The serum levels of lactate were measured using commercial kits. $n=6$ mice per group. Data are presented as the means \pm SEM. ${ }^{*} \mathrm{P}<0.05$ vs. the vehicle-treated sham. ${ }^{*} \mathrm{P}<0.05$ vs. the CLP+2-DG group. CLP, cecal ligation and puncture; i.p., intraperitoneally; 2-DG, 2-deoxy-D-glucose; PBS, phosphate-buffered saline; PDK1, pyruvate dehydrogenase kinase 1; PKM2, pyruvate kinase M2; LDHA, lactate dehydrogenase A.

2-DG (Fig. 5B). In the present study, LPS increased the apoptosis of HK-2 cells compared to the control group, and 2-DG significantly decreased this increase of apoptosis in HK-2 cells stimulated with LPS (Fig. 5C). These results indicated that 2-DG further upregulated autophagy in HK-2 cells stimulated with LPS.

Aerobic glycolysis inhibitor 2-DG increases autophagy via the SIRT3/AMPK pathway in septic mice and in $\mathrm{HK}-2$ cells stimulated with LPS. The mechanism of modulation of autophagy by 2-DG was further investigated by assessing the levels of autophagy regulators SIRT3 and AMPK in the kidney tissues of sepsis-induced AKI and HK-2 cells. p-AMPK and SIRT3 were evaluated by western blotting. The results revealed that the expression levels of SIRT3 and p-AMPK were upregulated in the kidney tissue of septic mice compared to the sham group, and 2-DG further increased SIRT3 and p-AMPK expression in septic mice (Fig. 6A and B). Concurrently it was revealed that LPS promoted the expression of SIRT3 and p-AMPK in HK-2 cells compared to the control group, and 2-DG further enhanced the expression of SIRT3 and
p-AMPK in HK-2 cells stimulated with LPS (Fig. 6C and D). These results indicated that $2-\mathrm{DG}$ increased autophagy via the SIRT3/AMPK pathway in sepsis-induced AKI.

Glycolysis metabolite lactate decreases autophagy via the SIRT3/AMPK pathway in HK-2 cells stimulated with LPS. Metabolism provides energy and intermediate metabolites may serve as signaling molecules to regulate cellular immune functions (22). Lactic acid is one of the most important metabolites of the glycolysis pathway. In the present study it was revealed that lactate treatment reduced the ratio of LC3-II/I and increased the expression of p62 in LPS-stimulated HK-2 cells compared with LPS group (Fig. S1A-C). Concurrently, lactate treatment decreased the expression of SIRT3 and p-AMPK in LPS-stimulated HK-2 cells compared with the LPS group (Fig. S1A, D and E). Furthermore, lactate partly abolished the increased effect on the ratio of LC3II/I and the decreased effect of p62 by 2-DG in LPS-treated HK-2 cells (Fig. 7A and B). Lactate treatment also decreased the increased effect on the expression of SIRT3 and p-AMPK by 2-DG in LPS-treated HK-2 cells (Fig. 7C and D). In addition, 

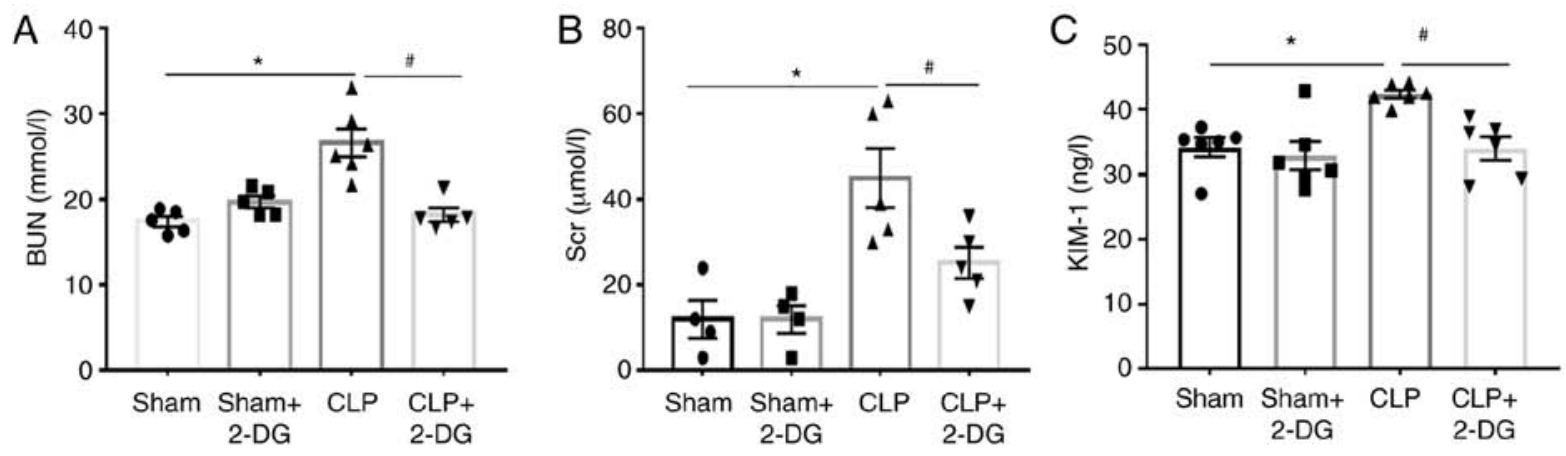

D

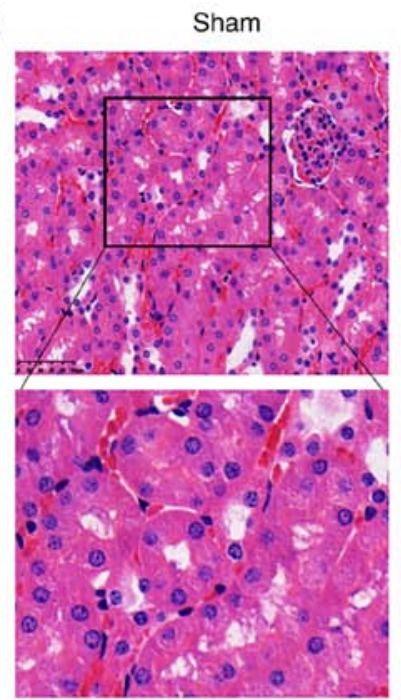

Sham+2-DG
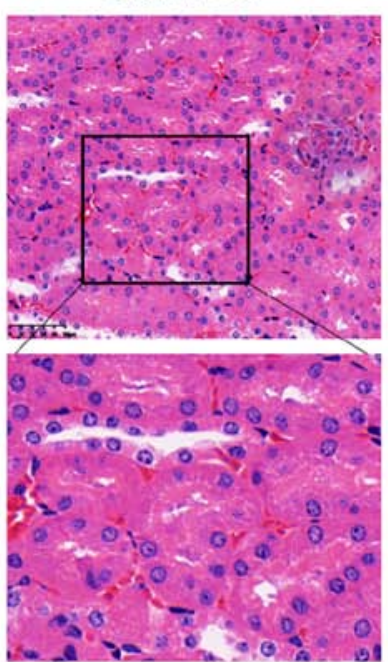

CLP
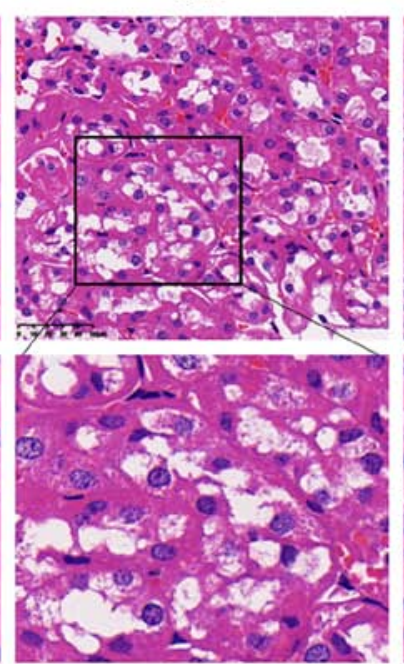

$C L P+2-D G$

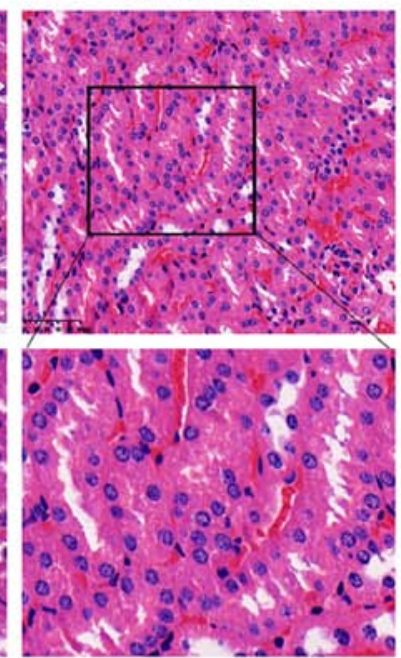

Figure 2. Glycolysis inhibitor 2-DG alleviates sepsis-induced AKI and apoptosis. Sepsis was induced in BALB/c mice by CLP. BALB/c mice were injected i.p. with either 2-DG (2 g/kg) or PBS in equivalent volumes $3 \mathrm{~h}$ before CLP. Sham-operated animals served as negative controls. At $24 \mathrm{~h}$ of CLP or sham operation, serum and kidneys were harvested from mice. (A-C) The levels of BUN, SCr and KIM-1 in mouse sera were analyzed using commercial kits. $\mathrm{n}=4-6$ mice per group. (D) The effect of 2-DG on the morphological changes of kidney tissues in CLP or sham mice (x40). Data are presented as the means \pm SEM. ${ }^{*} \mathrm{P}<0.05$ vs. the vehicle-treated sham. ${ }^{\#} \mathrm{P}<0.05$ vs. CLP+2-DG group. 2-DG, 2-deoxy-D-glucose; AKI, acute kidney injury; CLP, cecal ligation and puncture; i.p., intraperitoneally; PBS, phosphate-buffered saline; BUN, blood urea nitrogen; Scr, serum creatinine; KIM-1, kidney injury molecule-1.

\section{A}

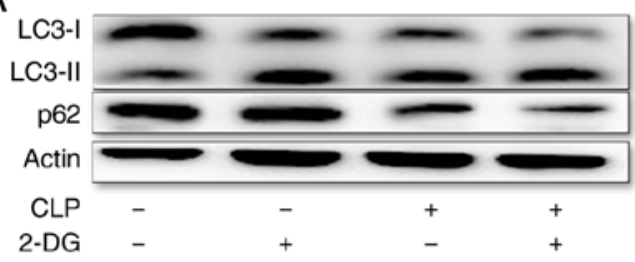

C

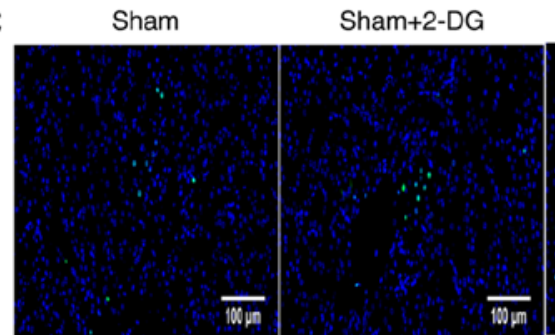

B

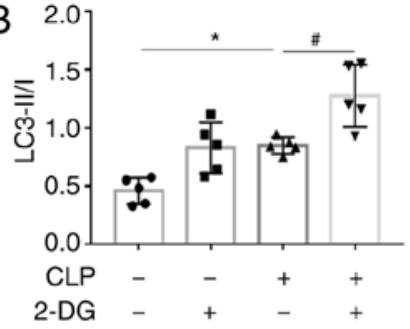

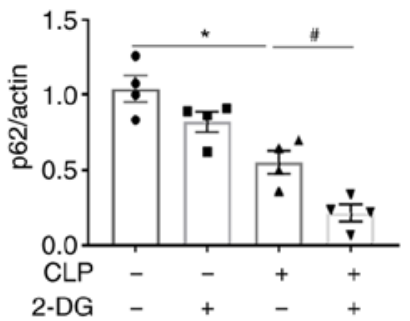

CLP+2-DG

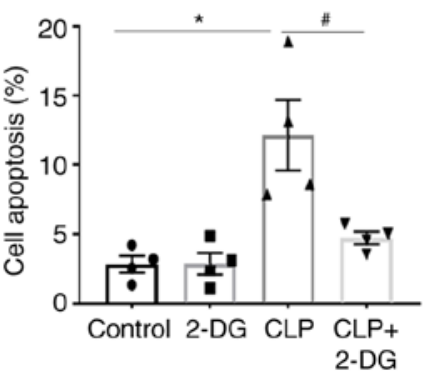

Figure 3. Glycolysis inhibitor 2-DG induces autophagy in kidney tissues of septic mice. Sepsis was induced in BALB/c mice by CLP. BALB/c mice were injected i.p. with either 2-DG $(2 \mathrm{~g} / \mathrm{kg})$ or PBS in equivalent volumes $3 \mathrm{~h}$ before CLP. Sham-operated animals served as negative controls. At $24 \mathrm{~h}$ of CLP or sham operation, kidney tissues were harvested from mice. (A and B) Western blotting of LC3-II/I and p62 in the whole kidney of septic mice. n=4 experiments. (C) The apoptosis of renal tubular cells of mice was measured by TUNEL assay and quantified. Scale bars, $100 \mu \mathrm{m}$. $\mathrm{n}=4 \mathrm{mice}$ per group. Data are presented as the means \pm SEM. ${ }^{*} \mathrm{P}<0.05$ vs. the vehicle-treated sham. ${ }^{\#} \mathrm{P}<0.05$ vs. the CLP+2-DG group. 2-DG, 2-deoxy-D-glucose; CLP, cecal ligation and puncture; i.p., intraperitoneally; PBS, phosphate-buffered saline; TUNEL, terminal UTP nick end labeling. 

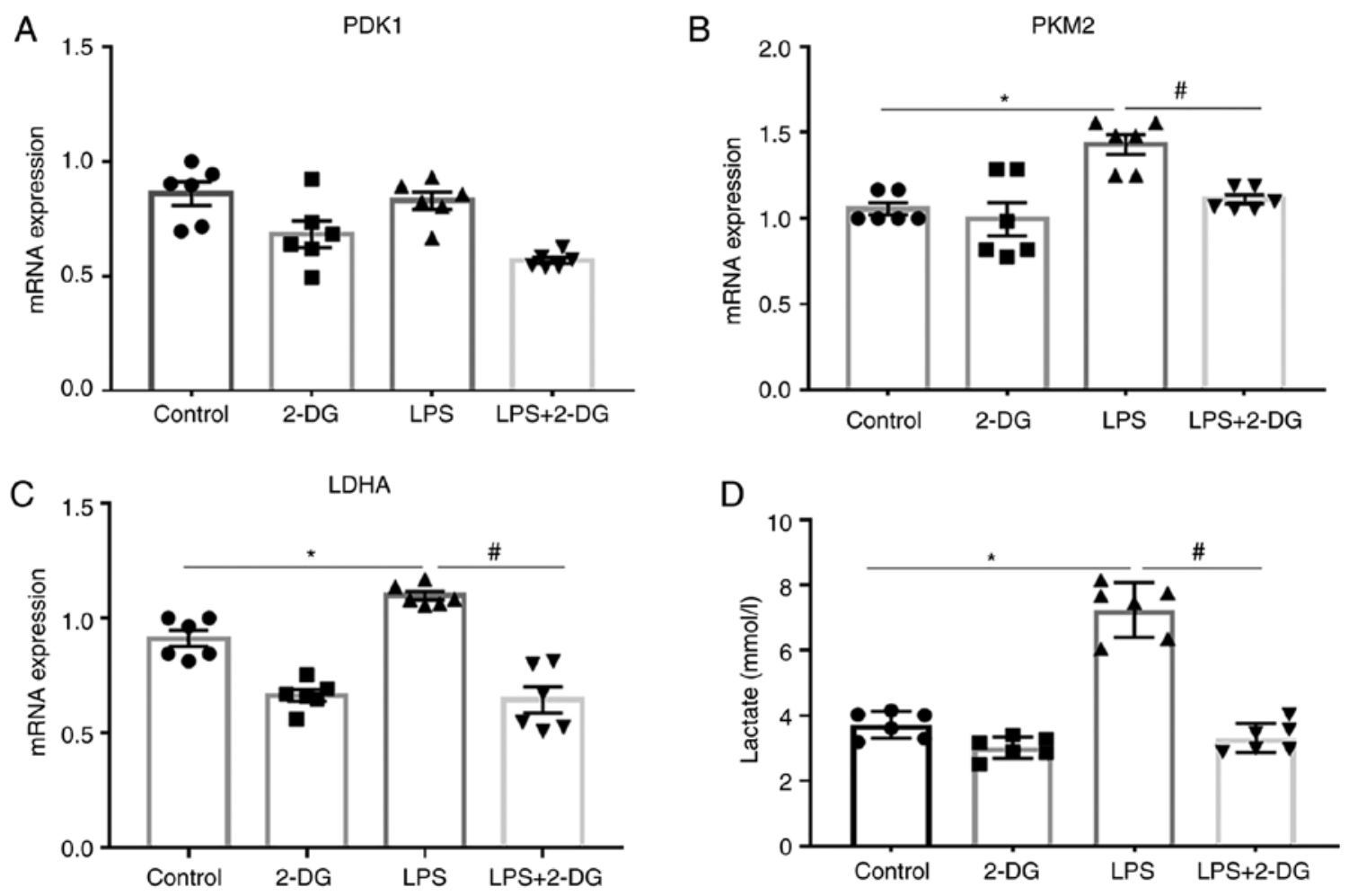

Figure 4. Assessment of glycolysis in HK-2 cells stimulated with LPS. A total of $1 \times 10^{6} \mathrm{HK}-2$ cells were treated with the doses of LPS $(1 \mu \mathrm{g} / \mathrm{ml}), 2-\mathrm{DG}(2 \mathrm{mM})$, and LPS+2-DG for $12 \mathrm{~h}$ at $37^{\circ} \mathrm{C}$ in 5\% $\mathrm{CO}_{2}$. (A-C) The mRNA expression of glycolysis-related genes (PDK1, PKM2 and LDHA) in HK-2 cells was analyzed by real-time PCR. $n=6$ samples per group. (D) The lactate levels in the supernatant of HK-2 cells were measured using commercial kits. $n=6$ samples per group. Data are presented as the means \pm SEM. ${ }^{*} \mathrm{P}<0.05$ vs. the control group. ${ }^{~} \mathrm{P}<0.05$ vs. the LPS +2 -DG group. LPS, lipopolysaccharides; 2-DG, 2-deoxy-D-glucose; PDK1, pyruvate dehydrogenase kinase 1; PKM2, pyruvate kinase M2; LDHA, lactate dehydrogenase A.
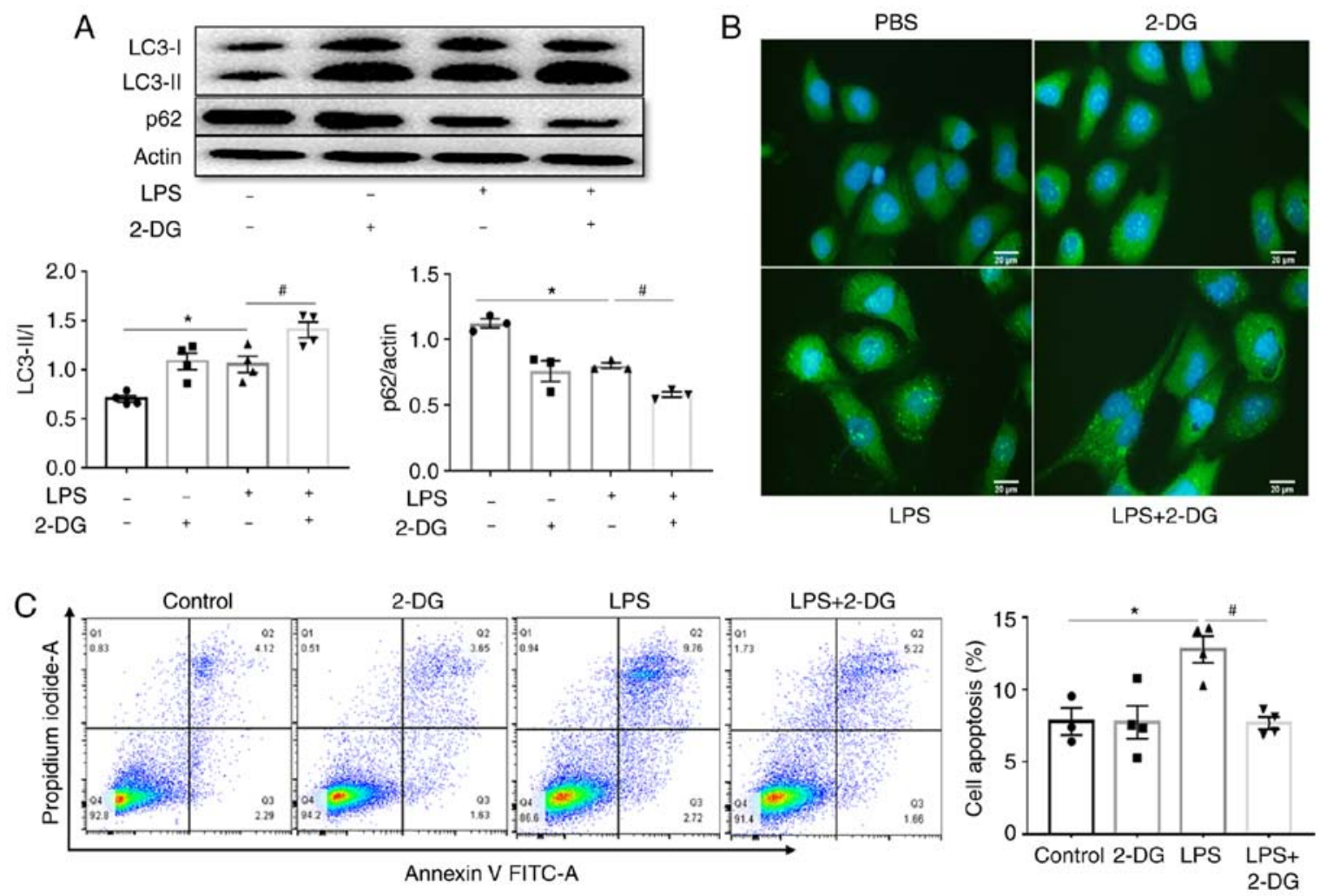

Figure 5. Glycolysis inhibitor 2-DG induces autophagy in HK-2 cells stimulated with LPS. A total of $1 \times 10^{6} \mathrm{HK}-2$ cells were treated with the doses of LPS $(1 \mu \mathrm{g} / \mathrm{ml}), 2-D G(2 \mathrm{mM})$, and LPS+2-DG for $12 \mathrm{~h}$ at $37^{\circ} \mathrm{C}$ in $5 \% \mathrm{CO}_{2}$. (A) Western blotting of LC3-I/II and p62 in HK-2 cells. $\mathrm{n}=4$ experiments. (B) Immunofluorescence staining of LC3 (green) expression in HK-2 cells. Scale bars, $20 \mu \mathrm{m}$. (C) The apoptosis of HK-2 cells was detected by flow cytometry. $\mathrm{n}=4$ samples per group. Data are presented as the means $\pm \mathrm{SEM}$. ${ }^{*} \mathrm{P}<0.05$ vs. the control group. ${ }^{*} \mathrm{P}<0.05$ vs. the LPS+2-DG group. 2-DG, 2-deoxy-D-glucose; LPS, lipopolysaccharides. 

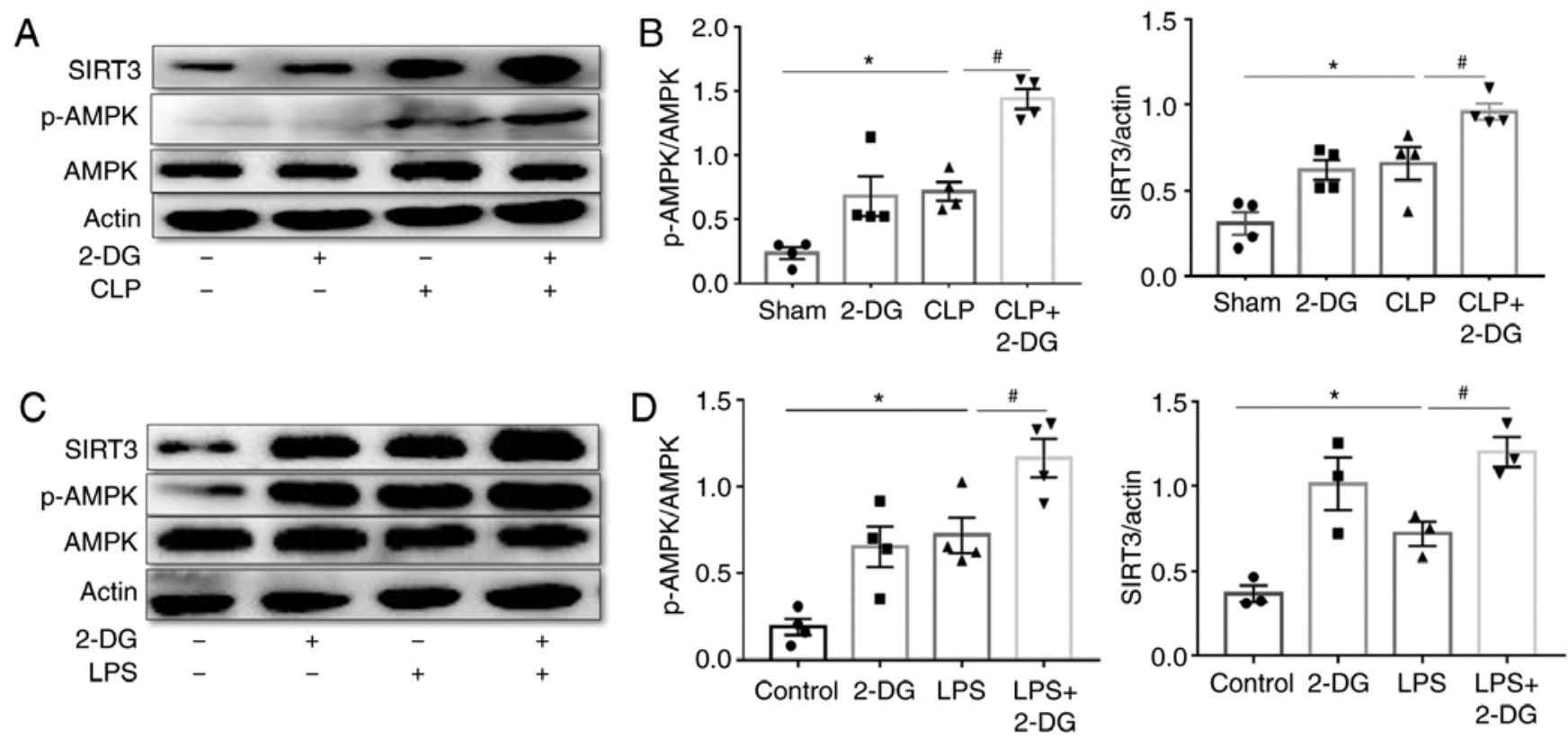

Figure 6. Glycolysis inhibitor 2-DG induces autophagy by regulating the SIRT3/AMPK pathway. Sepsis was induced in BALB/c mice by CLP. BALB/c mice were injected i.p. with either 2-DG $(2 \mathrm{~g} / \mathrm{kg})$ or PBS in equivalent volumes $3 \mathrm{~h}$ before CLP. Sham-operated animals served as negative controls. At $24 \mathrm{~h}$ of CLP or sham operation, kidneys were harvested from mice. (A and B) Immunoblot analysis and quantification of SIRT3 and p-AMPK/AMPK in the kidney tissues of septic mice in the presence/absence of 2-DG $(2 \mathrm{~g} / \mathrm{kg}) . \mathrm{n}=4$ experiments. (C and D) Immunoblot analysis and quantification of SIRT3 and p-AMPK/AMPK expression in HK-2 cells stimulated with LPS $(1 \mu \mathrm{g} / \mathrm{ml})$ for $12 \mathrm{~h}$ in the presence/absence of 2 -DG $(2 \mathrm{mM})$. $\mathrm{n}=4$ experiments. Data are presented as the means \pm SEM. ${ }^{*} \mathrm{P}<0.05$ vs. the vehicle-treated sham group or control group. ${ }^{*} \mathrm{P}<0.05$ vs. CLP+2-DG or LPS+2-DG group. 2-DG, 2-deoxy-D-glucose; SIRT3, sirtuin 3; AMPK, AMP-activated protein kinase; CLP, cecal ligation and puncture; i.p., intraperitoneally; PBS, phosphate-buffered saline; p-, phosphorylated; LPS, lipopolysaccharides.

lactate treatment reversed the reduction in apoptosis induced by $2-\mathrm{DG}$ in LPS-treated HK-2 cells (Fig. 7E). These results indicated that the glycolysis metabolite lactate was involved in regulating autophagy via the SIRT3/AMPK pathway.

2-DG-induced protective effects against sepsis-induced AKI depend on regulating autophagy. Finally, the involvement of autophagy in the protective effect of 2-DG on AKI was further examined using the autophagy inhibitor 3-MA. Inhibition of autophagy by 3-MA suppressed the upregulation of LC3II and the decrease of $\mathrm{p} 62$ by 2-DG in the kidney tissues of septic mice (Fig. 8A and B). 3-MA partly abolished the decreases in BUN, Scr and KIM-1 levels by 2-DG in septic mice (Fig. 8C and D). It was determined that 3-MA partially abrogated the decrease in apoptosis induced by 2-DG in the kidney tissues of septic mice (Fig. 8E). Furthermore, similar results were obtained with HK-2 cells. 3-MA suppressed the increase in LC3II and the reduction in p62 by 2-DG in LPS-treated HK-2 cells (Fig. S2A and B). 3-MA also partially abrogated the decreased effect of 2-DG on apoptosis in HK-2 cells stimulated with LPS (Fig. S2C and D). These results indicated that 2-DG-mediated protection against sepsis-induced AKI was related to the regulation of autophagy.

\section{Discussion}

In our previous study, we revealed that inhibition of aerobic glycolysis played a protective role in sepsis by promoting neutrophil migration into the infectious site, and we determined that treatment with the glycolysis inhibitor 2-DG improved the survival rates and attenuated sepsis-induced kidney injury (16). In the present study, we further examined the mechanisms underlying the protective effect of the inhibition of aerobic glycolysis on sepsis-induced AKI by focusing on the potential involvement and modulation of autophagy by SIRT3 and AMPK. AKI was induced by CLP in BALB/C mice, and examination of serum and kidney tissues and assessment of autophagy modulated by activation of SIRT3 and AMPK, revealed the protective effects of 2-DG, on kidney function and structure.

Aerobic glycolysis (Warburg effect), first observed in various tumor cells, is also increasingly recognized as an essential regulator of the immune response in both immune and nonimmune cells and represents a novel target for inflammatory diseases (23). Previous studies revealed that patients with AKI converted metabolism from oxidative phosphorylation to aerobic glycolysis $(24,25)$. Our group as well as other researchers revealed that experimental sepsis induces a shift of metabolism towards aerobic glycolysis in renal tissue. In a rodent model of CLP-induced sepsis, metabolomics analysis demonstrated an increase in the level of glycolytic intermediates concomitant with a decrease in flux through the tricarboxylic acid cycle (13). In the present study, it was revealed that sepsis enhanced aerobic glycolysis as evidenced by increased production of lactate and upregulated mRNA expression of glycolysis-related genes in kidney tissues in vivo. 2-DG markedly reduced this effect in vivo. This is consistent with previous studies $(13,24,25)$. In addition, HK-2 cells stimulated with LPS were used to establish a cell model of AKI. It was also determined that LPS enhanced aerobic glycolysis as evidenced by increased lactate production and upregulated mRNA expression of glycolysis-related genes in HK-2 cells 
A
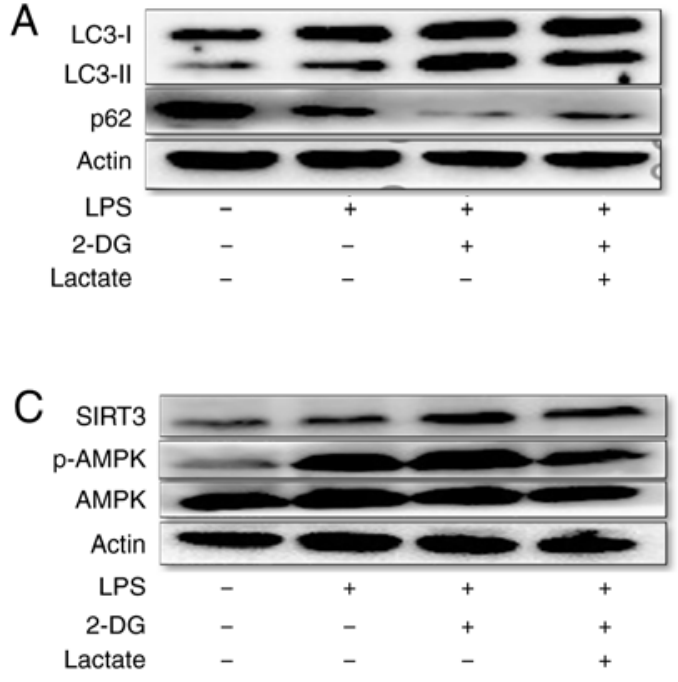
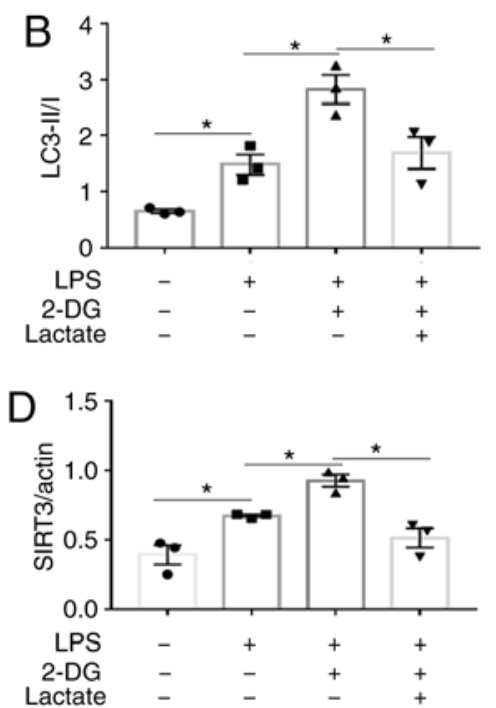
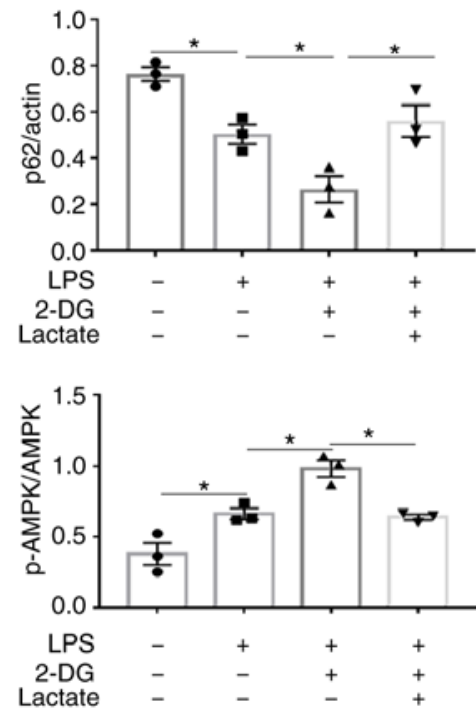
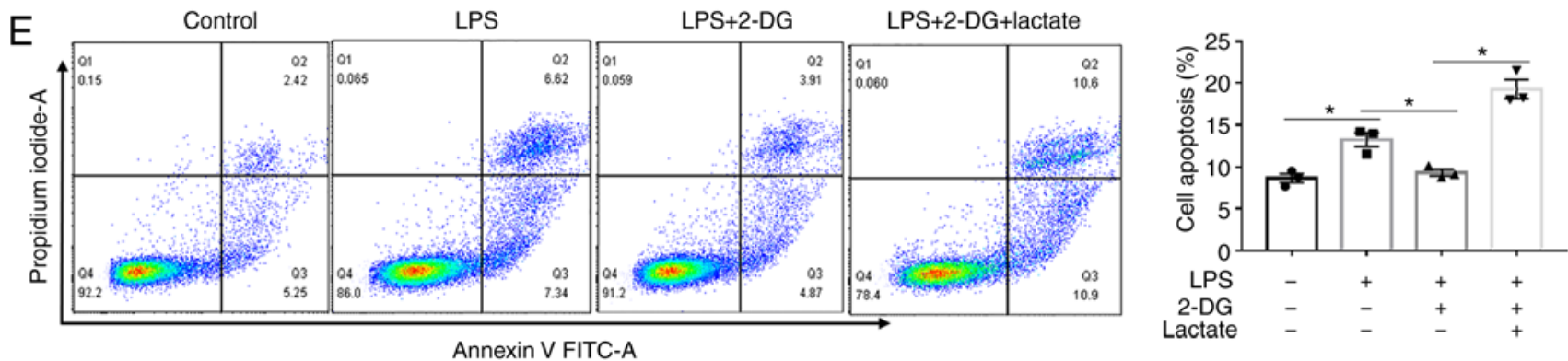

Figure 7. Lactate decreases autophagy via the SIRT3/AMPK pathway in HK-2 cells. A total of $1 \times 10^{6} \mathrm{HK}-2$ cells were treated with the indicated doses of LPS $(1$ $\mu \mathrm{g} / \mathrm{ml})$, LPS+2-DG $(2 \mathrm{mM})$, LPS $+2-\mathrm{DG}+$ lactate $(25 \mu \mathrm{M})$ for $12 \mathrm{~h}$ at $37^{\circ} \mathrm{C}$ in $5 \% \mathrm{CO}_{2}$. (A and B) Immunoblot analysis and quantification of LC3-I/II and p62 in HK-2 cells stimulated with LPS $(1 \mu \mathrm{g} / \mathrm{ml})$ in the presence/absence of lactate $(25 \mu \mathrm{M})$ or 2 -DG $(2 \mathrm{mM}$, before $3 \mathrm{~h}) . \mathrm{n}=3$ mice per group. (C and D) Immunoblot analysis and quantification of SIRT3 and p-AMPK/AMPK in HK-2 cells stimulated with LPS in the presence/absence of lactate (25 $\mu$ M) or 2-DG (2 mM, before $3 \mathrm{~h}$ ). $\mathrm{n}=4$ experiments. (E) Apoptosis of HK-2 cells stimulated with LPS in the presence/absence of lactate $(25 \mu \mathrm{M})$ or 2 -DG $(2 \mathrm{mM}$, before $3 \mathrm{~h})$. $\mathrm{n}=3$ experiments. The data are presented as the means \pm SEM. ${ }^{*} \mathrm{P}<0.05$, vs. the indicated groups. SIRT3, sirtuin 3; AMPK, AMP-activated protein kinase; LPS, lipopolysaccharides; 2-DG, 2-deoxy-D-glucose; p-, phosphorylated.

in vitro, which could be reduced by 2-DG. These data indicated that tubular epithelial cells (TECs) undergo similar metabolic changes in response to inflammation and suggest that aerobic glycolysis is upregulated in sepsis-induced AKI.

Studies have identified that aerobic glycolysis (namely the Warburg effect) is involved in the regulation of innate immune function and is a novel target of kidney disease $(11,26)$. Chiaravalli et al and Riwanto et al revealed that inhibition of aerobic glycolysis with 2-DG greatly reduced disease progression and improved the function of the kidney in polycystic kidney disease $(27,28)$. Numerous studies have demonstrated that a switch to aerobic glycolysis may be harmful to sepsis with evidence that stimulating the promoters of OXPHOS protected organs from damage and improved survival in experimental sepsis $(11,16,27,28)$. Treatment with a PKM2 inhibitor or knockdown of PKM2 significantly decreased glycolysis and reduced mortality in CLP-induced septic mice (13). Our group as well as other researchers verified that inhibition of aerobic glycolysis improved the survival rate and protected against multiorgan injury in sepsis $(16,18)$. Early reprogramming of metabolic pathways is not only able to protect the kidney from further injury, but also determines the fate of tissue repair and the progression to fibrosis and chronic organ dysfunction in sepsis-induced AKI $(10,11)$. In the present study, it was revealed that inhibition of aerobic glycolysis had a protective effect on sepsis-induced AKI with evidence of decreased BUN and Scr levels and apoptosis of tubular epithelial cells. Therefore, interventions designed to regulate the metabolism of the immune system may have potential therapeutic significance for sepsis-induced AKI.

Autophagy is a process of lysosome-mediated degradation of intracellular organelles, proteins, and other macromolecules $(29,30)$, which is detected at low levels in the normal physiological situation, but it can be significantly activated under starvation, hypoxia, and infection (31). The dysregulation of autophagy pathways has been implicated in the pathogenesis of numerous renal diseases including AKI, polycystic kidney disease, and diabetic nephropathy $(32,33)$. Numerous studies have revealed that autophagy is activated in renal tubular epithelial cells upon the occurrence of AKI, and activation of autophagy has a protective effect on the AKI model $(3,5,34)$. In addition, immune metabolism plays a critical role in modulating autophagy. Some studies have verified that knockdown of PKM2 decreases glycolysis and induces autophagy $(15,35)$. In the present study, it was determined that CLP upregulated the autophagy marker, LC3-II and decreased p62, while 2-DG further induced autophagy in CLP mice in parallel with decreased levels of BUN, Scr and 

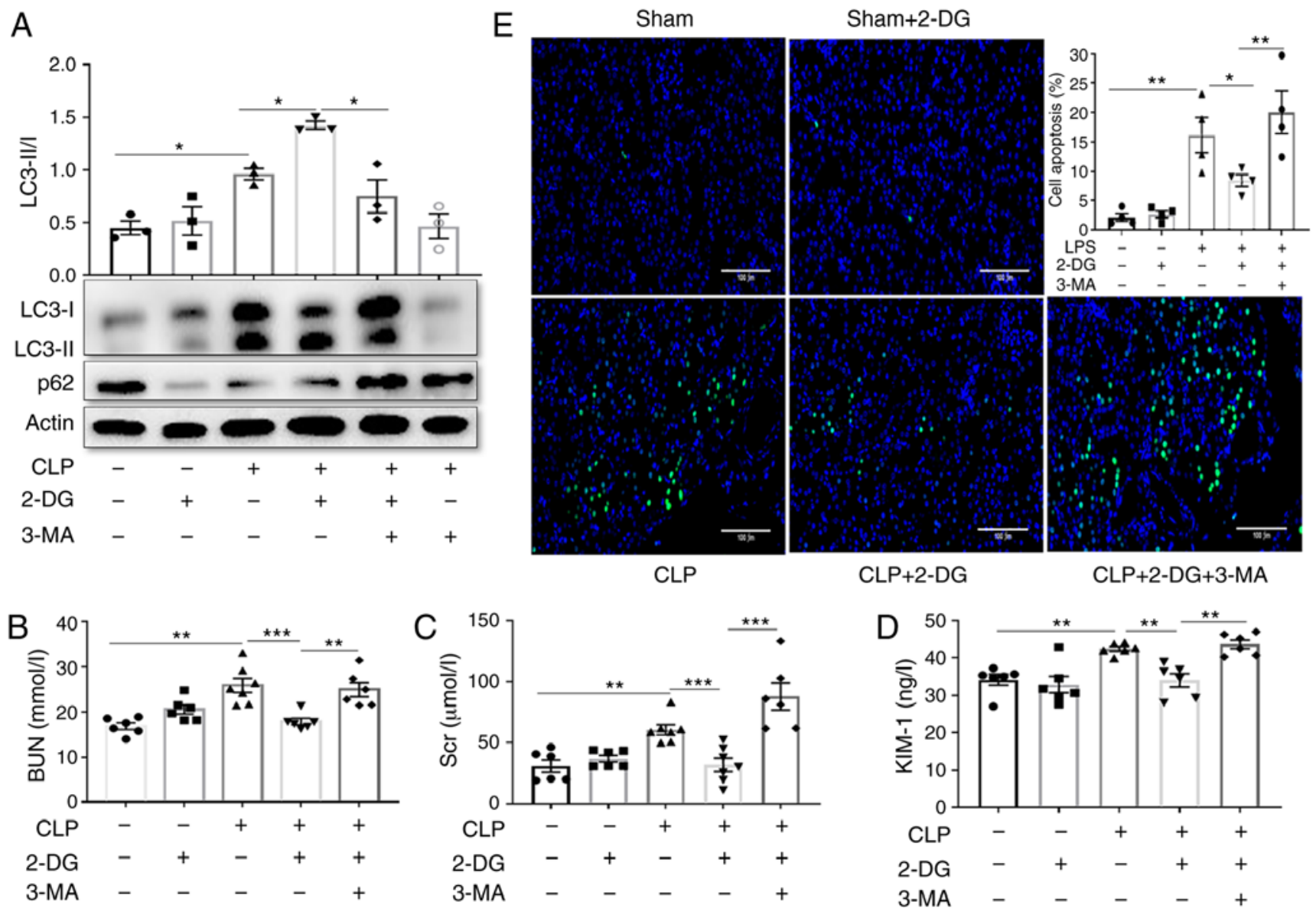

Figure 8. 2-DG-induced protective effects against sepsis-induced AKI depend on regulating autophagy. Mice were injected i.p. with either 3-MA (30 mg/kg, CLP+3-MA) or PBS in equivalent volumes $1 \mathrm{~h}$ before CLP in the presence/absence of 2-DG ( $\mathrm{g} / \mathrm{kg}$, before $3 \mathrm{~h})$. Kidney tissues and blood samples were collected at $24 \mathrm{~h}$ after CLP. (A) Immunoblot analysis and quantification of LC3-I/II and in kidney tissue of septic mice. $\mathrm{n}=3$ mice per group. (B-D) BUN and Scr and KIM-1 were analyzed using commercial kits. $n=7$ mice per group. (E) Apoptosis of renal tubular cells of mice was measured by TUNEL assay and quantified. Scale bars, $100 \mu \mathrm{m} . \mathrm{n}=4$ mice per group. Data are presented as the means $\pm \mathrm{SEM}$. ${ }^{*} \mathrm{P}<0.05,{ }^{* *} \mathrm{P}<0.01$ and ${ }^{* * * *} \mathrm{P}<0.001$ vs. the indicated groups. 2 -DG, 2-deoxy-D-glucose; AKI, acute kidney injury; i.p., intraperitoneally; 3-MA, 3-methyladenine; CLP, cecal ligation and puncture; PBS, phosphate-buffered saline; BUN, blood urea nitrogen; Scr, serum creatinine; KIM-1, kidney injury molecule-1; TUNEL, terminal UTP nick end labeling.

KIM-1, which indicated renal dysfunction. Similar results were obtained in LPS-treated HK-2 cells where 2-DG also upregulated autophagy. However, inhibition of autophagy by 3-MA suppressed the upregulation of LC3II and the decrease in 662 by 2-DG in the kidney tissues of septic mice. Similar results were obtained in LPS-treated HK-2 cells. Moreover, 3-MA partly abolished the decreases in the BUN, Scr and KIM-1 levels induced by 2-DG in septic mice. According to the present results, LPS enhanced aerobic glycolysis and autophagy in vivo and in vitro concurrently, while inhibition of aerobic glycolysis by 2-DG further increased autophagy in LPS-treated HK-2 cells. It is inferred that LPS enhanced autophagy via other signaling pathways, and this effect was stronger than the inhibitory effect by LPS-increased aerobic glycolysis on autophagy, and thus inhibition of aerobic glycolysis by 2-DG further increased autophagy. All the aforementioned results emphasized the importance of our findings in elucidating a potential mechanism underlying the protective effect of inhibition of aerobic glycolysis against AKI, which is related to an augmented regulation of autophagy.

However, the mechanism by which aerobic glycolysis regulates autophagy in sepsis-induced AKI in HK-2 cells is unclear. Numerous studies have demonstrated that SIRT3 and AMPK regulated autophagy in AKI $(19,36)$. It has been reported that SIRT3 knockdown can reduce AMPK phosphorylation and significantly inhibit the activation of AMPK kinase (37). SIRT3 deletion inhibited autophagy in CLP mice in parallel with increased levels of BUN and Scr, which indicated renal dysfunction. These effects were accompanied by downregulation of p-AMPK and upregulation of p-mTOR (36). In the present study, western blotting and quantification of the results revealed that treatment with 2-DG induced autophagy in parallel with upregulation of SIRT3 and p-AMPK in septic mice. Based on the aforementioned results, it was concluded that autophagy was associated with SIRT3/AMPK, in the CLP mouse model. Concurrently, the same results were also obtained in HK-2 cells. It was revealed that 2-DG further induced autophagy and increased SIRT3 and p-AMPK in HK-2 cells stimulated with LPS. In addition, glycolysis has been reported to regulate autophagy as evidenced by 2-DG enhancement of autophagy through activation of the AMPK/ULK1 pathway and in cancer cells (38). Modulation of sepsis-enhanced glycolysis with 2-DG improved the survival outcome by decreasing MKK3 phosphorylation and increasing SIRT3 expression (18). 
Moreover, Jin et al recently revealed that renal AMPK activation protected from sepsis-induced AKI by preserving mitochondrial function and metabolic fitness likely through SIRT3 signaling (39). The aforementioned results are consistent with the results of the present study. It was concluded that 2-DG decreased aerobic glycolysis and increased autophagy through the SIRT3/AMPK pathway, and protected against sepsis-induced AKI.

Lactic acid is one of the most important metabolites of the glycolysis pathway. Lactate is an immunosuppression factor and high serum lactic acid levels are a strong negative prognostic factor in severe septic patients (40). In the present study upregulated production of lactate in the serum of septic mice was observed. Lactate may be a potential and critical contributory factor in the regulation of immune function in sepsis. It has been reported that increased production of lactate suppressed autophagy in cancer cells (41). In the present study, it was revealed that lactate treatment decreased the autophagy marker LC3-II and increased p62 in the HK-2 cells stimulated with LPS. However, the molecular mechanism for the suppression of autophagy by lactate remains unclear. It was revealed that lactate treatment decreased p-AMPK and SIRT3 in LPS-treated HK-2 cells. Lactate also partly abolished the increased effect on LC3II/I and p62 by $2-\mathrm{DG}$ in LPS-treated HK-2 cells. Notably, Xu et al already demonstrated that lower $\mathrm{pH}$ and higher $\mathrm{pH}$ conditions induced completely opposite effects on autophagy, with its activity suppressed at lower $\mathrm{pH}$ whereas stimulated at higher $\mathrm{pH}$ (42). It is not certain whether lactate-suppressed autophagy is a direct effect of lactate or an indirect effect of extracellular $\mathrm{pH}$, further research is required to confirm this hypothesis.

In conclusion, the present study revealed a potential mechanism underlying the protective role of inhibition of aerobic glycolysis against sepsis-induced AKI, which was involved in the induction of autophagy through the lactate/SIRT3/AMPK pathway. These findings suggest that the reprogramming of the metabolism is important in renal injury and is a potential therapeutic target of AKI.

\section{Acknowledgements}

The authors are grateful to Dr Kewen Ma (Department of Pathology, Xiangya Hospital, Central South University, Changsha, China) for her help with the analysis of the morphological tubular H\&E dye in this study. We also thank the associate editor and the reviewers for their useful feedback that improved this study.

\section{Funding}

The present study was supported by funding from the National Natural Science Foundation of China, grant nos. 81871610 , 81870071,81471897 and 81671895.

\section{Availability of data and materials}

The datasets generated during the present study are not currently available to the public but will be available from the corresponding author on reasonable request.

\section{Authors' contributions}

XX and HZ oversaw the study. XX and CT designed and conceived the study. CT, JG, TL, HC, and KL collected samples, performed experiments, and analyzed data. CT and ML prepared the figures and wrote the manuscript. $\mathrm{HZ}$ and $\mathrm{XX}$ reviewed the manuscript for important intellectual content. All authors read and approved the manuscript and agree to be accountable for all aspects of the research in ensuring that the accuracy or integrity of any part of the work are appropriately investigated and resolved.

\section{Ethics approval and consent to participate}

All animal experimental protocols were approved by the Institutional Animal Care and Use Committee of Central South University (approval no. 2019sydw0027) and performed according to the guidelines of the Ethics Committee of the Animal Experimental Institute of Central South University, Changsha, China.

\section{Patient consent for publication}

Not applicable.

\section{Competing interests}

The authors declare that they have no competing interests.

\section{References}

1. Uchino S, Kellum JA, Bellomo R, Doig GS, Morimatsu H, Morgera S, Schetz M, Tan I, Bouman C, Macedo E, et al: Acute renal failure in critically ill patients: A multinational, multicenter study. JAMA 294: 813-818, 2005.

2. Murugan R and Kellum JA: Acute kidney injury: What's the prognosis? Nat Rev Nephrol 7: 209-217, 2011.

3. Cruz MG, Dantas JG, Levi TM, Rocha Mde S, de Souza SP, Boa-Sorte N, de Moura CG and Cruz CM: Septic versus non-septic acute kidney injury in critically ill patients: Characteristics and clinical outcomes. Rev Bras Ter Intensiva 26: 384-391, 2014 (In En, Portuguese)

4. Mehta RL, Bouchard J, Soroko SB, Ikizler TA, Paganini EP, Chertow GM and Himmelfarb J; Program to Improve Care in Acute Renal Disease (PICARD) Study Group: Sepsis as a cause and consequence of acute kidney injury: Program to improve care in acute renal disease. Intensive Care Med 37: 241-248, 2011.

5. Kaushal GP and Shah SV: Autophagy in acute kidney injury. Kidney Int 89: 779-791, 2016.

6. Duann P, Lianos EA, Ma J and Lin PH: Autophagy, innate immunity and tissue repair in acute kidney injury. Int J Mol Sci 17: 662,2016

7. Jiang M, Wei Q, Dong G, Komatsu M, Su Y and Dong Z: Autophagy in proximal tubules protects against acute kidney injury. Kidney Int 82: 1271-1283, 2012.

8. Li T, Liu Y, Zhao J, Miao S, Xu Y, Liu K, Liu M, Wang G and Xiao X: Aggravation of acute kidney injury by mPGES-2 down regulation is associated with autophagy inhibition and enhanced apoptosis. Sci Rep 7: 10247, 2017.

9. Liu S, Hartleben B, Kretz O, Wiech T, Igarashi P, Mizushima N, Walz G and Huber TB: Autophagy plays a critical role in kidney tubule maintenance, aging and ischemia-reperfusion injury. Autophagy 8: 826-837, 2012.

10. Zager RA: 'Biologic memory' in response to acute kidney injury: Cytoresistance, toll-like receptor hyper-responsiveness and the onset of progressive renal disease. Nephrol Dial Transplant 28: 1985-1993, 2013.

11. Gomez H, Kellum JA and Ronco C: Metabolic reprogramming and tolerance during sepsis-induced AKI. Nat Rev Nephrol 13: 143-151, 2017. 
12. Smith JA, Stallons LJ and Schnellmann RG: Renal cortical hexokinase and pentose phosphate pathway activation through the EGFR/Akt signaling pathway in endotoxin-induced acute kidney injury. Am J Physiol Renal Physiol 307: F435-F444, 2014

13. Waltz P, Carchman E, Gomez H and Zuckerbraun B: Sepsis results in an altered renal metabolic and osmolyte profile. J Surg Res 202: 8-12, 2016.

14. Yang J, Zhou R and Ma Z: Autophagy and energy metabolism. Adv Exp Med Biol 1206: 329-357, 2019.

15. Chu B, Wang J, Wang Y and Yang G: Knockdown of PKM2 induces apoptosis and autophagy in human A549 alveolar adenocarcinoma cells. Mol Med Rep 12: 4358-4363, 2015.

16. Tan C, Gu J, Chen H, Li T, Deng H, Liu K, Liu M, Tan S, Xiao Z, Zhang $\mathrm{H}$ and Xiao X: Inhibition of aerobic glycolysis promotes neutrophil to influx to the infectious site via CXCR2 in sepsis. Shock 53: 114-123, 2020.

17. Hubbard WJ, Choudhry M, Schwacha MG, Kerby JD, Rue LW III Bland KI and Chaudry IH: Cecal ligation and puncture. Shock 24 (Suppl 1): S52-S57, 2005.

18. Zheng Z, Ma H, Zhang X, Tu F, Wang X, Ha T, Fan M, Liu L, $\mathrm{Xu} \mathrm{J}, \mathrm{Yu} \mathrm{K}$, et al: Enhanced glycolytic metabolism contributes to cardiac dysfunction in polymicrobial sepsis. J Infect Dis 215 1396-1406, 2017

19. Zhao W, Zhang L, Chen R, Lu H, Sui M,Zhu Y and Zeng L: SIRT3 protects against acute kidney injury via AMPK/mTOR-regulated autophagy. Front Physiol 9: 1526, 2018.

20. Livak KJ and Schmittgen TD: Analysis of relative gene expression data using real-time quantitative PCR and the 2(-Delta Delta C(T)) method. Methods 25: 402-408, 2001.

21. Mariño G, Niso-Santano M, Baehrecke EH and Kroemer G: Self-consumption: The interplay of autophagy and apoptosis. Nat Rev Mol Cell Biol 15: 81-94, 2014.

22. Ganeshan K and Chawla A: Metabolic regulation of immune responses. Annu Rev Immunol 32: 609-634, 2014.

23. Palsson-McDermott EM and O'Neill LA: The Warburg effect then and now: From cancer to inflammatory diseases. Bioessays 35: 965-973, 2013.

24. Bar-Or D, Carrick M, Tanner A II, Lieser MJ, Rael LT and Brody E: Overcoming the Warburg effect: Is it the key to survival in sepsis? J Crit Care 43: 197-201, 2018.

25. Xie M, Yu Y, Kang R, Zhu S, Yang L, Zeng L, Sun X, Yang M, Billiar TR, Wang H, et al: PKM2-dependent glycolysis promotes NLRP3 and AIM2 inflammasome activation. Nat Commun 7 13280,2016

26. Rowe I, Chiaravalli M, Mannella V, Ulisse V, Quilici G, Pema M, Song XW, Xu H, Mari S, Qian F, et al: Defective glucose metabolism in polycystic kidney disease identifies a new therapeutic strategy. Nat Med 19: 488-493, 2013.

27. Chiaravalli M, Rowe I, Mannella V, Quilici G, Canu T, Bianchi V, Gurgone A, Antunes S, D'Adamo P, Esposito A, et al: 2-Deoxy-d-glucose ameliorates PKD progression. J Am Soc Nephrol 27: 1958-1969, 2016.

28. Riwanto M, Kapoor S, Rodriguez D, Edenhofer I, Segerer S and Wuthrich RP: Inhibition of aerobic glycolysis attenuates disease progression in polycystic kidney disease. PLoS One 11: e0146654, 2016
29. Mizushima N and Komatsu M: Autophagy: Renovation of cells and tissues. Cell 147: 728-741, 2011.

30. Huber TB, Edelstein CL, Hartleben B, Inoki K, Jiang M, Koya D, Kume S, Lieberthal W, Pallet N, Quiroga A, et al: Emerging role of autophagy in kidney function, diseases and aging. Autophagy 8: 1009-1031, 2012.

31. Rubinsztein DC, Codogno P and Levine B: Autophagy modulation as a potential therapeutic target for diverse diseases. Nat Rev Drug Discov 11: 709-730, 2012.

32. Kume S, Thomas MC and Koya D: Nutrient sensing, autophagy, and diabetic nephropathy. Diabetes 61: 23-29, 2012.

33. Belibi F, Zafar I, Ravichandran K, Segvic AB, Jani A, Ljubanovic DG and Edelstein CL: Hypoxia-inducible factor-1a (HIF-1 $\alpha$ ) and autophagy in polycystic kidney disease (PKD). Am J Physiol Renal Physiol 300: F1235-F1243, 2011.

34. Zhang Y, Wang L, Meng L, Cao G and Wu Y: Sirtuin 6 overexpression relieves sepsis-induced acute kidney injury by promoting autophagy. Cell Cycle 18: 425-436, 2019.

35. Dey P, Kundu A, Sachan R, Park JH, Ahn MY, Yoon K, Lee J, Kim ND, Kim IS, Lee BM and Kim HS: PKM2 knockdown induces autophagic cell death via AKT/mTOR pathway in human prostate cancer cells. Cell Physiol Biochem 52: $1535-1552,2019$

36. Li S, Dou X, Ning H, Song Q, Wei W, Zhang X, Shen C, Li J, Sun $C$ and Song Z: Sirtuin 3 acts as a negative regulator of autophagy dictating hepatocyte susceptibility to lipotoxicity. Hepatology 66: 936-952, 2017.

37. Shi T, Fan GQ and Xiao SD: SIRT3 reduces lipid accumulation via AMPK activation in human hepatic cells. J Dig Dis 11: 55-62, 2010.

38. Li W, Tanikawa T, Kryczek I, Xia H, Li G, Wu K, Wei S, Zhao L, Vatan L, Wen B, et al: Aerobic glycolysis controls myeloid-derived suppressor cells and tumor immunity via a specific CEBPB isoform in triple-negative breast cancer. Cell Metab 28: 87-103.e106, 2018.

39. Jin K, Ma Y, Manrique-Caballero CL, Li H, Emlet DR, Li S, Baty CJ, Wen X, Kim-Campbell N, Frank A, et al: Activation of AMP-activated protein kinase during sepsis/inflammation improves survival by preserving cellular metabolic fitness. FASEB J 34: 7036-7057, 2020.

40. Nolt B, Tu F, Wang X, Ha T, Winter R, Williams DL and Li C: Lactate and immunosuppression in sepsis. Shock 49: 120-125, 2018.

41. Im JH, Kang KW, Kim SY, Kim YG, An YJ, Park S, Jeong BH, Choi SY, Lee JS and Kang KW: GPR119 agonist enhances gefitinib responsiveness through lactate-mediated inhibition of autophagy. J Exp Clin Cancer Res 37: 295, 2018

42. Xu T, Su H, Ganapathy S and Yuan ZM: Modulation of autophagic activity by extracellular pH. Autophagy 7: 1316-1322, 2011.

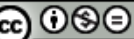

This work is licensed under a Creative Common Attribution-NonCommercial-NoDerivatives 4.0 International (CC BY-NC-ND 4.0) License. 